\title{
Cerebellar hemangioblastoma mimicking an aneurysm: A case report and literature review
}

\author{
YU-CHEN JI $^{1 *}$, YAN LI $^{2 *}$, JING-XIA HU $^{2}$, HONG-BO ZHANG $^{3}$, PENG-XIANG YAN ${ }^{4}$ and HUAN-CONG ZUO ${ }^{2}$ \\ ${ }^{1}$ Department of Neurosurgery, First Affiliated Hospital of Zhengzhou University, Zhengzhou 450052; \\ ${ }^{2}$ Department of Neurosurgery, Tsinghua University Yuquan Hospital, Beijing 100040; \\ ${ }^{3}$ Department of Neurosurgery, Xinhua Hospital of Hubei, Wuhan, Hubei 430015; \\ ${ }^{4}$ Department of Neurosurgery, Beijing Tiantan Hospital, Capital Medical University, Beijing 100050, P.R. China
}

Received April 15, 2015; Accepted July 15, 2016

DOI: $10.3892 / \mathrm{ol} .2016 .4979$

\begin{abstract}
Hemangioblastomas (HBMs) are highly vascular tumors of the central nervous system. Sporadic HBMs are nearly always solitary, and solitary HBMs are similar to intracranial arteriovenous malformations due to their highly vascular characteristics. However, to the best of our knowledge, cases of HBM in the cerebellum mimicking an aneurysm have never been reported in the literature. The present study reports a case of an HBM on the right cerebellar hemisphere mimicking an aneurysm, which originated from the right posterior inferior cerebellar artery, as determined using magnetic resonance angiography and digital subtraction angiography. The patient was admitted the Department of Neurosurgery at the Tsinghua University Yuquan Hospital (Beijing, China) in January 2015 due to a 4-year history of intermittent headaches. The diagnosis of an HBM was determined during surgery and the tumor was totally resected by changing the operation technique, with no complications. In conclusion, it is difficult to distinguish between HBMs and intracranial vascular diseases, particularly aneurysms. Surgeons should consider the possibility carefully prior to surgery and careful prepare for each eventuality.
\end{abstract}

\section{Introduction}

Hemangioblastomas (HBMs) are highly vascular tumors of the central nervous system, which represent $1.5-2.5 \%$ of all intracranial tumors (1). HBMs may occur sporadically in the central nervous system or in association with von Hippel-Lindau disease (2). Morphologically, HBMs appear as solid, solid-cystic or mainly cystic with a small mural, vascularized

Correspondence to: Professor Peng-Xiang Yan, Department of Neurosurgery, Beijing Tiantan Hospital, Capital Medical University, 6 Tiantan Xili, Dongcheng, Beijing 100050, P.R. China

E-mail: yanneurosuegeon@126.com

${ }^{*}$ Contributed equally

Key words: hemangioblastoma, cerebellar, aneurysm nidus. Sporadic HBMs are nearly always solitary, due to their highly vascular nature. The characteristics of solid variants of HBMs, including a clear vascular blush, multiple feeding vessels and draining veins on digital subtraction angiography (DSA) (3), are similar to intracranial arteriovenous malformations (AVMs) $(4,5)$, and to the best of our knowledge, a case of an HBM in the cerebellum mimicking an aneurysm have never been reported in the literature. The present study reports a case of a cerebellar HBM mimicking an aneurysm that was treated via surgical resection. The patient provided written informed consent for publication of this case report and any accompanying images.

\section{Case report}

A 52-year-old man presented with a 4-year history of intermittent headaches at the Tsinghua University Yuquan Hospital (Beijing, China) in January 2015. No other nervous system deficits were found. Brain computed tomography (Fig. 1) and magnetic resonance imaging (MRI) (Fig. 2) revealed a $0.9 \times 0.9-\mathrm{cm}$ mass on the right cerebellar hemisphere and showed significant contrast enhancement. MR angiography demonstrated a significant vascular blush supplied from the right posterior inferior cerebellar artery (PICA) (Fig. 3). All these results were consistent with an aneurysm. Lateral vertebral artery DSA then showed a highly vascular tumor nodule supplied by the right PICA (Fig. 4), so a diagnosis of an aneurysm originating from the PICA was considered.

The patient was scheduled to undergo a suboccipital retrosigmoid craniotomy, however, during the procedure the suspected aneurysm was found to be an HBM and the surgical strategy was changed. A total resection of the tumor was performed without any complications. The tumor was pathologically confirmed as HBM due to the results of a histological examination of the excised mass. Briefly, resected tissues were fixed in formalin, parrafin-embedded and cut into $4-\mu \mathrm{m}$ sections, prior to staining with hematoxylin and eosin and visualization under a light microscope. The histopathological analysis revealed that the tumor was composed of stromal cells and abundant vascular endothelial cells (Fig. 5). Follow-up MRI performed at 1 and 6 months post-surgery showed no evidence of recurrence. 


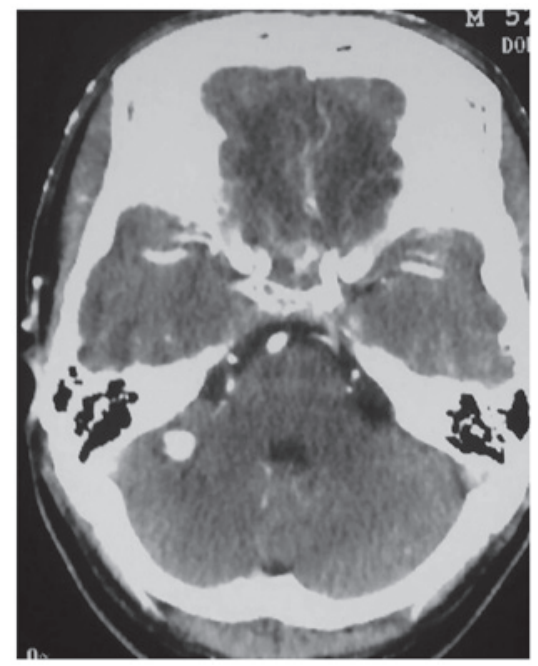

Figure 1. Contrast-enhanced computed tomography revealing a homogeneous mass on the right cerebellar hemisphere.

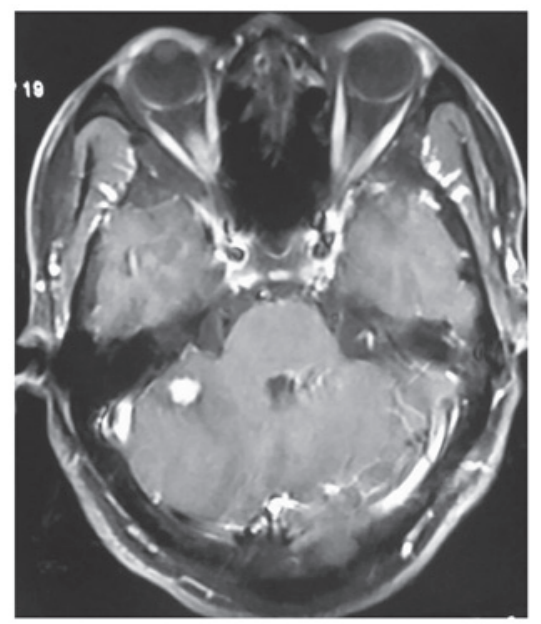

Figure 2. Contrast enhanced magnetic resonance imaging scan showing a mass on the right cerebellar hemisphere.

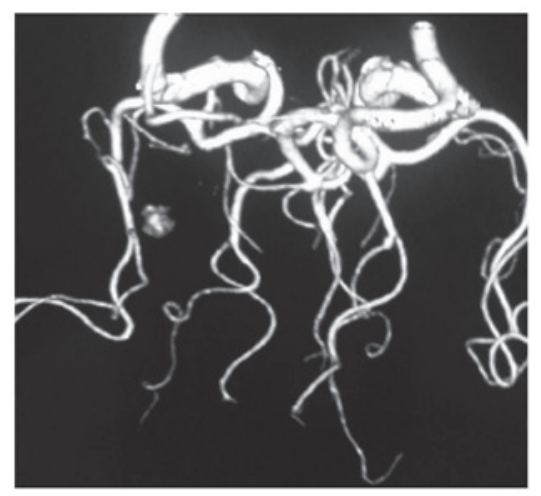

Figure 3. Magnetic resonance angiography demonstrating significant vascular blush supplied mostly from the right posterior inferior cerebellar artery.

\section{Discussion}

According to the World Health Organization (6), HBMs are classified as grade I tumors, with a highly vascularized solid

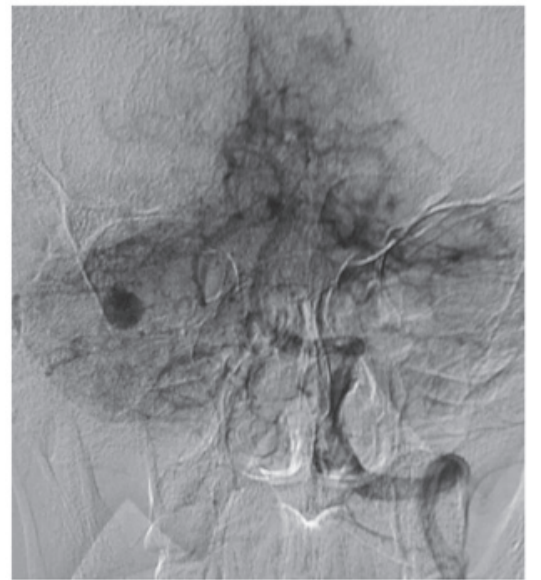

Figure 4 . Vertebral artery digital subtraction angiography showing a highly vascular tumor nodule supplied by the right posterior inferior cerebellar artery.

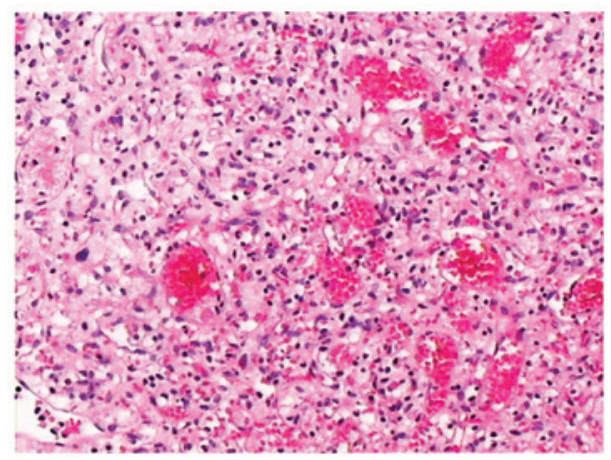

Figure 5. Hematoxylin and eosin staining of the resected mass revealing that the hemangioblastoma was composed of stromal cells and abundant vascular endothelial cells (magnification, x100).

tumor component. The tumors mainly occur in the cerebellar hemisphere, spinal cord and brain stem (7), but are rarely found in the supratentorial region (8) due to its high degree of vascularity. HBMs are easily misdiagnosed as AVMs or intracranial aneurysms.

HBMs that appear as mainly cystic with a small nodule are diagnosed easily due to their typical imaging characteristic. However, solid HBMs present as round-shaped masses, which are iso- or hypodense on T1-weighted images and hyperdense on T2-weighted images, together with a significant contrast enhancement pattern. Serpiginous flow voids are found on T1- and T2-weighted images, which are characteristic of solid HBMs (9). It is crucial to form a differential diagnosis between solid HBM and vascular diseases pre-operatively, particularly between AVM and aneurysm. An AVM nidus presents as a dense vascular network similar to an HBM nodule. However, HBMs may cause mass effects, which leads to neurological symptoms. Moreover, HBMs present with a microvascular structure that is similar to a normal capillary bed, whereas an AVM nidus is formed from altered vessels with no actual capillary bed (10). HBMs may coexist with intracranial aneurysms due to hemodynamic stress mechanisms. Therefore, an intracranial aneurysm should also be considered when hemorrhage caused by HBM occurs (11). DSA is necessary for 
the differential diagnosis of HBM, since it permits the identification of the feeding artery and enlargement of draining veins (12). As was shown in the present case, the characteristics on DSA are similar to an aneurysm originating from the PICA, therefore, a tumor may wrongly be considered as an aneurysm prior to surgery.

Surgical resection is the major treatment for HBMs due to their benign features. However, surgical treatment is often challenging, particularly for solid tumors with a highly vascularized nature, and the mortality associated with the biopsy or removal of a solid HBM is high. Therefore, internal decompression and any attempt at biopsy or partial resection should be avoided (13). With the progression of microsurgical techniques, it is now feasible to remove these tumors totally (3). The successful resection of a solid HBM should follow the principles of an AVM dissection. This means that feeding arteries should be identified and blocked first, followed by tumor dissection and occlusion of the draining veins. As the premature obliteration of the venous drainage can result in terminal intraoperative swelling and hemorrhage, a circumferential dissection with devascularization and en bloc removal is favored whenever possible in solid tumors. Importantly, all surgical instruments, including those for the alternative procedure, should be made ready pre-operatively. The surgical strategy should then be changed when the misdiagnosis is identified intraoperatively.

Debate remains over whether the pre-operative embolization of an HBM should be performed. It has been suggested that the pre-operative embolization of an HBM can facilitate surgical removal and allow complete tumor resection by significantly reducing the tumor blood supply (14), controlling the inaccessible artery supply and reducing the vascularity of the tumor (15). Occasionally, pre-operative embolization may significantly avoid profuse intraoperative bleeding. However, it has also been reported that pre-operative embolization using particles has a high risk for acute tumor bleeding and mortality (16). In our opinion, the pre-operative embolization of HBM can reduce the risk of intraoperative bleeding and shorten the surgical duration, which may result in the improved success of a tumor resection and reduced surgical complications.

The effectiveness of radiotherapy or chemotherapy in HBM has been questioned (17-19). Although certain studies have demonstrated that pre-operative radiotherapy can control local tumor progression and finally facilitate complete and safe tumor removal $(4,20)$, the long-term results of radiosurgery require confirmation (18). Therefore, we believe that radiotherapy or chemotherapy should only be used for residual or recurrent tumors post-operatively.

Solid HBMs are easily misdiagnosed as AVMs or intracranial aneurysms due to their high degree of vascularity. DSA is necessary to make the differential diagnosis and microsurgical resection is the treatment of choice due to the benign features of the disease. Pre-operative embolization can increase the surgical effectiveness and reduce the complications. Assistant therapies should only be used for residual or recurrent tumors after microsurgery.

In conclusion, the present study described the case of a 52-year-old male patient who presented with HBM mimicking an aneurysm on DSA preoperatively, which was confirmed as HBM during surgery via a histopathological analysis. The tumor was totally resected according to the principles of an AVM dissection, and no recurrence was found in follow-up MRI. This case demonstrates that HBM may be easily misdiagnosed as an aneurysm.

\section{References}

1. Resche F, Moisan JP, Mantoura J, de Kersaint-Gilly A, Andre MJ, Perrin-Resche I, Menegalli-Boggelli D, Lajat Y and Richard S: Haemangioblastoma, haemangioblastomatosis, and von Hippel-Lindau disease. Adv Tech Stand Neurosurg 20: 197-304, 1993.

2. Conway JE, Chou D, Clatterbuck RE, Brem H, Long DM, Rigamonti D: Hemangioblastomas of the central nervous system in von Hippel-Lindau syndrome and sporadic disease. Neurosurgery 48: 55-62; discussion 62-63.

3. Rachinger J, Buslei R, Prell J and Strauss C: Solid haemangioblastomas of the CNS: A review of 17 consecutive cases. Neurosurg Rev 32: 37-47, 2009.

4. Kamitani H, Hirano N, Takigawa H, Yokota M, Miyata H, Ohama E and Watanabe T: Attenuation of vascularity by preoperative radiosurgery facilitates total removal of a hypervascular hemangioblastoma at the cerebello-pontine angle: Case report. Surg Neurol 62: 238-243; discussion 243-244, 2004.

5. Dow GR, Sim DW and O'Sullivan MG: Excision of large solid haemangioblastomas of the cerebellopontine angle by a skull base approach. Br J Neurosurg 16: 168-171, 2002.

6. Louis DN, Ohgaki H, Wiestler OD, Cavenee WK, Burger PC, Jouvet A, Scheithauer BW and Kleihues P: The 2007 WHO Classification of Tumours of the Central Nervous System. Acta Neuropathol 114: 97-109, 2007.

7. Hussein MR: Central nervous system capillary haemangioblastoma: The pathologist's viewpoint. Int J Exp Pathol 88: 311-324, 2007.

8. Kim H, Park IS and Jo KW: Meningeal supratentorial hemangioblastoma in a patient with von hippel-lindau disease mimicking angioblastic menigioma. J Korean Neurosurg Soc 54: 415-419, 2013.

9. Gelabert González M: Posterior fossa hemangioblastomas. Neurologia 22: 853-859, 2007 (In Spanish).

10. de San Pedro JR, Rodríguez FA, Níguez BF, Sánchez JF, López-Guerrero AL, Murcia MF and Vilar AM: Massive hemorrhage in hemangioblastomas literature review. Neurosurg Rev 33: 11-26, 2010.

11. Suzuki M, Umeoka K, Kominami S and Morita A: Successful treatment of a ruptured flow-related aneurysm in a patient with hemangioblastoma: Case report and review of literature. Surg Neurol Int 5 (Suppl 9): S430-S433, 2014.

12. Takeuchi S, Tanaka R, Fujii Y, Abe H and Ito Y: Surgical treatment of hemangioblastomas with presurgical endovascular embolization. Neurol Med Chir (Tokyo) 41: 246-252, 2001.

13. Okawara SH: Solid cerebellar hemangioblastoma. J Neurosurg 39: 514-518, 1973.

14. Dabus G, Pryor J, Spilberg G, Samaniego EA and Nogueira RG: Embolization of intra-axial hypervascular tumors with Onyx: Report of three cases. J Neurointerv Surg 5: 177-180, 2013.

15. Standard SC, Ahuja A, Livingston K, Guterman LR and Hopkins LN: Endovascular embolization and surgical excision for the treatment of cerebellar and brain stem hemangioblastomas. Surg Neurol 41: 405-410, 1994.

16. Cornelius JF, Saint-Maurice JP, Bresson D, George B and Houdart E: Hemorrhage after particle embolization of hemangioblastomas: Comparison of outcomes in spinal and cerebellar lesions. J Neurosurg 106: 994-998, 2007.

17. Capitanio JF, Mazza E, Motta M, Mortini P and Reni M: Mechanisms, indications and results of salvage systemic therapy for sporadic and von Hippel-Lindau related hemangioblastomas of the central nervous system. Crit Rev Oncol Hematol 86: 69-84, 2013.

18. Niemelä M, Lim YJ, Söderman M, Jääskeläinen J and Lindquist C: Gamma knife radiosurgery in 11 hemangioblastomas. J Neurosurg 85: 591-596, 1996.

19. Pan L, Wang EM, Wang BJ, Zhou LF, Zhang N, Cai PW and Da JZ: Gamma knife radiosurgery for hemangioblastomas. Stereotact Funct Neurosurg 70 (Suppl 1): S179-S186, 1998.

20. Moss JM, Choi CY, Adler JJ Jr, Soltys SG, Gibbs IC and Chang SD: Stereotactic radiosurgical treatment of cranial and spinal hemangioblastomas. Neurosurgery 65: 79-85, 2009. 